\title{
Stress Behavior in Premature Infants with Periventricular Leukomalacia
}

\author{
Hirotaka Gima ${ }^{1,3)}$, Shohei Ohgi ${ }^{2)}$, TAKayuki Fujiwara ${ }^{1)}$, Koji AbE ${ }^{3)}$ \\ 1) Koriyama Institute of Health Sciences: 2-9-3 Zukei, Koriyama-city, Fukushima 963-8834, \\ Japan.TEL: +81 24-936-7784, FAX: +81 24-936-7778,E-mail: h-gima@k-tohto.ac.jp \\ 2) Department of Rehabilitation, Seirei Christopher University \\ ${ }^{3)}$ Interdisciplinary Graduate School of Science and Technology, Shinshu University
}

J. Phys. Ther. Sci.

22: 109-115, 2010

\begin{abstract}
Purpose] The aim of this study was to evaluate the stress behavior of infants with preventricular leukomalacia (PVL) using direct behavioral observation. [Subjects] The participants were 15 infants with and 73 infants without PVL. [Methods] This research examined infants' stress behaviors in response to handling during a Brazelton Neonatal Behavioral Assessment Scale (NBAS) exam. Observation of the stress behaviors was recorded at the bedside. Infants' behaviors were simultaneously videotaped. The assessment was performed at the postconceptional age of 38-40 weeks (term). [Results] The with-PVL group showed significantly higher frequency of stress behaviors in the autonomic (pale/cyanotic, tremor, startle, twitch), motor (arm flexion, legs extension, jerky movements, finger splay, arching, salute, hand clasp, foot clasp, holding on, flaccidity, grimacing) and state-orientation (fuss, panicked or worried alertness, gaze aversion, high pitched cry) subsystems compared with the without-PVL group. [Conclusion] Behavioral observations may help clinicians assess preterm infant neurological status and provide useful information for facilitating the development and adaptability of infants' behavioral organization.
\end{abstract}

Key words: Preventricular leukomalacia (PVL), Stress behavior, Behavioral observation

(This article was submitted Nov. 10, 2009, and was accepted Dec. 3, 2009)

\section{INTRODUCTION}

Developments in medical technology have markedly improved the survival rate of premature and low birth-weight infants. Nevertheless, such infants remain at increased risk of developmental difficulties. The rate of later cerebral palsy is 25 to 31 times higher among infants who weigh less than $1500 \mathrm{~g}$ at birth than among full-sized newborns ${ }^{1)}$. Babies with birth weights less than 2,500 g account for about one third of all babies who later have signs of cerebral palsy ${ }^{2}$. Periventricular leukomalacia (PVL)is a major risk factor for cerebral palsy and other handicaps in premature infants ${ }^{3)}$.

Although the value of clinically identifying infants with brain injuries is recognized, it is also important to understand the neonatal neurobehavioral characteristics of such infants, so that an appropriate intervention plan for the developmental care of these at-risk infants may be formulated. In developmental care of premature and neonatal infants, it is a fundamental concept to lessen the stress of infants, and promote the systematization of neurobehavioral development.

Behavioral observation of neonatal/preterm infants provides a way to infer an infant's developmental goals and to assess an infant's current functional competence. Infants display reliably observable behaviors along the lines of the three main systems, the autonomic system, the motor system, and the state system with special emphasis on the emerging attention system. The 
autonomic system's behavioral stress signals include breathing patterns, color fluctuations, and visceral responses such as spitting up, gagging, hiccoughing, bowel movement strains, and actual defection, among others. The motor system's behavioral stress signals include muscle tone of the trunk, extremities, and face with good modulation, flaccidity or hypertonicity; as well as postures and movement patterns, such as finger splays, arching, grimacing, or tucking together, grasping, and others. Furthermore, the behavioral stress signals of an infant's state system, which defines an infant's level of awareness, include the infant's range of states such as sleeping, wakefulness, and aroused upset; the patterns of transition from state to state, and the robustness and modulation of each of the states. All these reliably observable behavioral stresses provide valuable information to the clinician and caregiver on how to structure and adapt care and interaction, in order to enhance an infant's own competencies, strength and signals of self regulation, well-being, and reaching out/ initiation, and to prevent or diminish an infant's signals of stress, discomfort ${ }^{4,5)}$.

Ohgi, et al. found that compared with infants without PVL in the neonatal period, infants with PVL showed dysfunction and/or disorganization of the neurobehavioral system as reflected by significantly lower NBAS scores (Habituation, Orientation, Motor, Range of State, State Regulation and Autonomic Stability) ${ }^{6}$. Although the value of clinically identifying infants with brain injuries is recognized, it is also important to understand the neonatal neurobehavioral characteristics of these infants, so that an appropriate intervention plan for the developmental care of these at-risk infants may be formulated. Assessment of infant behaviors could strengthen the nursing care and developmental intervention for preterm infants. However, there is little research validation of response differences to handling of preterm infants with and without PVL. The purpose of this study was to analyze the expression of the stress behaviors of infants with PVL based on the behavioral observations.

\section{SUBJECTS AND METHODS}

\section{Subjects}

This was a prospective study of low birth-weight and/or premature infants who were developmentally followed from birth for signs of PVL and motor disabilities at 12-18 months of age. Neurological examinations and observations of neurological signs were performed. Imaging by MRI and CT, and EEG were performed when an abnormality was observed in the neurological examination.

The study participants consisted of low birthweight and/or premature infants who were admitted to the Neonatal Intensive Care Unit (NICU) of the Nagasaki University Hospital, Nagasaki, Japan, between January 1998 and December 2001. During this period, 216 infants were treated; 78 of these infants were excluded: 7 died, 9 had congenital anomalies, 11 needed surgical treatment and 51 were discharged or transferred to the regular nursery within 2 weeks of birth. Among the 111 infants for whom we were able to give a prognostic diagnosis between 12 and 18 months of age, we diagnosed 15 as having PVL, 23 as having developmental disabilities, and 73 as healthy. The study participants were comprised of the $15 \mathrm{PVL}$ infants (with-PVL group) and the 73 healthy infants (without-PVL group) (Table1). Informed consent was provided by the participants' parents.

\section{Methods}

This research examined infant's stress behaviors in response to handling during a Brazelton Neonatal Behavioral Assessment Scale (NBAS) exam. Observation of the stress behaviors was recorded at the bedside using the Newborn Individualized Developmental Care and Assessment Program (NIDCAP) system. NIDCAP classifies infants' behavior into the autonomy, motor, and stateorientation subsystems. The NIDCAP methodology documents infants' continuous communications through the recording of detailed observation of infants' naturalistically occurring behaviors in the $\mathrm{NICU}^{7)}$. The naturalistic observation sheet (NIDCAP Sheet) provides a systematic format for the recording of the detailed observation of an individual infant's behavior every two minutes. Although NIDCAP is designed for behavioral observation with the infants in the incubator, this study was performed in the period when infants were able to be handled out of the incubator. In this study we observed infants behavior in response to handling using the Brazelton NBAS.

The NBAS was used for the infants' behavioral 
Table 1. Characteristics of study participants

\begin{tabular}{lccc}
\hline & with-PVL & without-PVL & $95 \%$ CI \\
\hline No. of subjects & 15 & 73 & \\
Sex (Male / Female) & $11 / 4$ & $44 / 29$ & $-4.4 \sim-1.9^{*}$ \\
Gestational age (week) & $30.0(25-36)$ & $33.0(27-39)$ & $-48.1 \sim-147.2$ \\
Birth weight (g) & $1360.0(680-2010)$ & $1631.0(905-2090)$ & \\
AGA / SGA infants & $13 / 2$ & $54 / 19$ & $-2.1 \sim-0.3^{* *}$ \\
Apgar score & $6(3-9)$ & $8(1-9)$ & $-2.0 \sim-0.3^{* *}$ \\
1 min & $8(1-10)$ & $9(1-10)$ & $1.7 \sim 10.6^{* *}$ \\
5 min $^{\dagger}$ & $7(46.7) *$ & $10(13.7)$ & $9.3 \sim 67.5^{*}$ \\
No. of intubation cases (\%) & $0(0-58)$ & $0(0-13)$ & $48(17-178)$ \\
Duration of intubation (day) ${ }^{\dagger}$ & $67(31-182)$ & & \\
Duration of hospital stay (day) & & &
\end{tabular}

AGA; appropriate for gestational age, SGA; small for gestational age, PVL; periventricular leukomalacia, $\mathrm{CI}$; cofidence interval. ${ }^{\dagger}$ Median (range), ${ }^{*} \mathrm{p}<0.05,{ }^{* *} \mathrm{p}<0.01$.

assessment to standardize the examination procedure. The NBAS has been the most consistently used assessment of neonatal neurobehavior in clinical practice and research ${ }^{8,9}$. The NBAS contains 28 behavioral items and 18 reflex items which are administered in a particular sequence and can be grouped into "packages" (Habituation, Motor-Oral, Trunk, Vestibular, Social-Interactive) which follow an established order. The order of items is organized according to their level of intensity or degree of stimulation. The initial items do not involve any handling of the baby, but as the examination progresses the items become more stimulating, beginning with minimally intrusive tactile items and ending with the more massive vestibular items. The NBAS was administered by examiners (author; SO) certified at the Brazelton Institute, to guarantee the validity and reliability of the examination. Infants' behaviors were simultaneously videotaped. Infants' stress behaviors were assessed through videotapes based on the NIDCAP observation models. The assessment was performed at the postconceptional age of 38-40 weeks (term).

The participants' characteristics were compared between the with and without-PVL groups using Pearson's chi-square test, the Mann-Whitney U test and Fisher's exact test depending on the distribution of each variable. The frequencies of each stress behavior were compared between the with and without-PVL groups using Pearson's chi-square. Logistic regression analysis was used to estimate the odds ratio (OR) of the stress behavior frequency of infants with PVL. Logistic regression analysis with two categories of dependent variable (with or without-PVL group) was performed. The independent variables included sex, birth weight, gestational age, AGA or SGA, and 5 minute Apgar score, because these factors had pronounced effects on infants' behavior. P values less than 0.05 (twotailed tests) were regarded as statistically significant. All statistical analyses were performed using SPSS for Windows (version 12.0J) software.

\section{RESULTS}

The characteristics of infants in each group are presented in Table 1. A comparison between the two groups revealed no significant differences in sex ratio, AGA or SGA status. In the with-PVL group, birth weight was significantly lighter, gestational age was significantly shorter, Apgar scores were significantly lower, and the duration of intubation and hospital stay, as well as requirement for mechanical ventilation were significantly longer compared to the without-PVL group.

Table 2 shows the results of the comparison of the frequency of autonomic stress behaviors between with- and without-PVL groups. Pearson's chisquare test showed there were significantly higher frequencies of Pale/Cyanotic, Tremor, Startle and Twitch in the with-PVL group. The estimated odds ratios of the with-PVL group associated with autonomic stress behavior calculated from logistic 
Table 2. Autonomic stress behavior

\begin{tabular}{lccc}
\hline & $\begin{array}{c}\text { with-PVL } \\
(\mathrm{n}=15)\end{array}$ & $\begin{array}{c}\text { without-PVL } \\
(\mathrm{n}=73)\end{array}$ & OR $(95 \% \mathrm{CI})^{\dagger}$ \\
\hline Respiratory pause & 0 & 0 & - \\
Tachypnea & 1 & 1 & - \\
Gasping & 0 & 0 & - \\
Pale/Cyanotic & 3 & 3 & $3.17(0.24-41.46)$ \\
Mottled/Webbed & 4 & 10 & $1.97(0.41-9.33)$ \\
Jaundiced & 0 & 0 & - \\
Gagging & 4 & 4 & $3.13(0.16-61.81)$ \\
Stipping up & 0 & 0 & - \\
Hiccoughing & 1 & 1 & $18.82(0.30-1193.69)$ \\
Tremors & $8 * *$ & 10 & $12.33(2.50-60.73)^{* *}$ \\
Startle & $11 * *$ & 11 & $13.46(2.70-67.17)^{* *}$ \\
Twitch & $1 *$ & 2 & $95.34(0.35-27767.63)$ \\
\hline
\end{tabular}

$* \mathrm{p}<0.05, * * \mathrm{p}<0.01$.

$\dagger$ Estimated odds ratios of the stress items for the with-PVL group compared to the withoutPVL group calculated from the logistic regression analysis.

regression analysis showed that the higher frequencies of Tremor and Startle were significantly associated with an increased risk of PVL.

Table 3 compares the frequencies of motor stress behaviors of the with- and without-PVL groups. Pearson's chi-square test showed there were significantly higher frequencies of Arm flexion, Legs extension, Jerky move, Finger splay, Arching, Salute, Hand clasp, Foot clasp, Holding on, Flaccidity and Grimacing in the with-PVL group. The estimated odds ratios of the with-PVL group associated with motor stress behavior calculated from logistic regression analysis showed that the higher frequencies of the Arm flexion, Legs extension, Jerky move, Arching, Hand clasp, Foot clasp, Holding on, Flaccidity and Grimacing were significantly associated with an increased risk of PVL.

Table 4 shows the frequencies of state-related stress behaviors of the with and without-PVL groups. Pearson's chi-square test showed there were significantly higher frequencies of the Fuss, Panicked or worried alertness, Gaze aversion and High pitched cry in the with-PVL group. The estimated odds ratios of with-PVL group associated with state related stress behavior calculated from logistic regression analysis showed that higher frequencies of Panicked or worried alertness and High pitched cry were significantly associated with an increased risk of PVL. Also, the number of state changes was significantly greater in with-PVL group than in the without-PVL group (with-PVL: median 7 (range 5-10), without-PVL; 5 (3-9) ).

\section{DISCUSSION}

Compared with the without-PVL group, autonomic, motor, and state-related stress behaviors occurred more frequently in the with-PVL group. Logistic regression analysis with adjustment of infants' background factors revealed that autonomic stress behaviors of Tremor and Startle were noted more frequently in the with-PVL group. Als et al. classified stress, an autonomy subsystem, into respiratory-circulatory system, digestive system, and nervous system factors ${ }^{7}$. Tremor and Startle are stress behaviors related to the nervous system. Our results suggest that higher frequencies of Tremor and Startle were signs of irritation of the central nervous system (CNS). No differences were found in stress behaviors related to the respiratorycirculatory system and the digestive system. Regulation of the respiratory-circulatory system tends to be observed at the gestational age of 24-30 weeks in premature infants ${ }^{10)}$, and physiological stress decreases over time until it stabilizes at 32 weeks ${ }^{11)}$. As participants in this study were at term age at the time of the NIDCAP behavioral observations, respiratory-circulatory system stresses were expected to have stabilized.

The observation of stress behavior of the motor system, the appearance rates of Arm flexion, Legs 
Table 3. Motor stress behavior

\begin{tabular}{lccc}
\hline & $\begin{array}{c}\text { with-PVL } \\
(\mathrm{n}=15)\end{array}$ & $\begin{array}{c}\text { without-PVL } \\
(\mathrm{n}=73)\end{array}$ & OR $(95 \% \mathrm{CI})^{\dagger}$ \\
\hline Arm flexion & $7^{* *}$ & 6 & $24.29(3.11-189.62)^{* *}$ \\
Legs flexion & 5 & 12 & $4.76(1.02-22.18)$ \\
Arms extension & 2 & 6 & $2.24(0.28-17.80)$ \\
Legs extension & $8^{* *}$ & 14 & $5.26(1.20-23.14)^{*}$ \\
Jerky move & $10^{* *}$ & 11 & $32.54(3.23-328.34)^{* *}$ \\
Finger splay & $6^{*}$ & 12 & $2.83(0.63-12.66)$ \\
Arching & $7^{* *}$ & 9 & $22.44(3.76-133.80)^{* *}$ \\
Sitting on air & $6^{*}$ & 20 & $1.44(0.36-5.84)$ \\
Salute & $4^{*}$ & 6 & $16.49(1.27-214.85)$ \\
Hand clasp & $6^{* *}$ & 9 & $22.88(3.24-161.38)^{* *}$ \\
Foot clasp & $6^{*}$ & 11 & $12.28(1.60-93.87)^{*}$ \\
Holding on & $6^{*}$ & 11 & $12.87(2.29-72.29)^{* *}$ \\
Flaccidity & $7^{* *}$ & 5 & $22.44(3.76-133.80)^{* *}$ \\
Grimacing & $11^{* *}$ & 11 & $32.28(4.97-209.65)^{* *}$ \\
Gape face & 4 & 9 & $1.57(0.30-8.33)$ \\
\hline
\end{tabular}

$* \mathrm{p}<0.05, * * \mathrm{p}<0.01$.

$\dagger$ Estimated odds ratios of the stress items for the with-PVL group compared to the without-PVL group calculated from the logistic regression analysis.

Table 4. State related stress behavior

\begin{tabular}{lccc}
\hline & with-PVL & without-PVL & OR $(95 \% \mathrm{CI})^{\dagger}$ \\
& $(\mathrm{n}=15)$ & $(\mathrm{n}=73)$ & \\
\hline Fuss & $7^{*}$ & 13 & $2.48(0.67-9.14)$ \\
Panicked or worried alertness & $5 * *$ & 5 & $8.16(1.36-49.17)^{*}$ \\
Face open & 0 & 2 & - \\
Gaze aversion & $4 *$ & 5 & $4.88(0.84-28.17)$ \\
Eye floating & 3 & 4 & $5.48(0.94-32.10)$ \\
Yawn & 2 & 10 & $0.60(0.07-5.42)$ \\
Sneeze & 1 & 6 & $1.11(0.10-12.78)$ \\
High piched cry & $3 * *$ & 3 & $17.18(1.42-208.34)^{*}$ \\
\hline
\end{tabular}

$* \mathrm{p}<0.05, * * \mathrm{p}<0.01$.

$\dagger$ Estimated odds ratios of the stress items for the with-PVL group compared to the without-PVL group calculated from the logistic regression analysis.

extension, Jerky move, Finger splay, Arching, salute, Hand clasp, Foot clasp, Holding on, Flaccidity, and Grimacing, was significantly higher in the with-PVL group. Stress signs of the motor system are also easily observed in premature infants at the gestational age of 24-30 weeks. Pressler et al. and Grunau et al. suggested that stress signs of the motor system arise from the organization of the neurobehavioral system accompanied by gestational age (maturity), disease, and environmental factors ${ }^{10,12)}$. Grunau et al. also suggested that grimacing, finger splay, legs extension and other disorganized motor behaviors in low birth weight infants are reactions to uncomfortable stimulations ${ }^{12,13)}$. Also, stress behaviors in the motor system are related to CNS dysfunction, and risk factors for brain injuries, such as PVL. Prechtl et al. and Cioni et al. suggested that evaluating GMs (general movements) of neonatal infants can provide an index of brain dysfunction ${ }^{14,15)}$. Assessment of the quality of infants' spontaneous movements can thus provide 
insights into the functional integrity of neonates CNS. There is empirical evidence to support the claim that the presence of markedly abnormal movements (i.e., GMs that are virtually devoid of complexity, variation and fluency) reflects the presence of serious brain dysfunction ${ }^{16-18)}$.

The frequencies of state-related stress behaviors such as Fuss, Panicked or worried Alertness, Gaze aversion, and High pitched cry were significantly higher in the with-PVL group than the without-PVL group. Also, the odds ratio of Panicked or worried alertness and High pitched cry were significantly higher in the with-PVL group. In addition, the times of state change (lability of state) in the withPVL group were significantly higher. Ohgi et al. has suggested that infants with PVL have characteristics of disorganized state regulation, such as easily state change, hypersensitivity to external stimulation and irritability ${ }^{6}$. Similar result was observed in this study.

Based on the Synactive Theory of Behavioral Organization developed by Als, infants' functioning occurs in a model of continuous intra-organism subsystem interactions. At each stage of development, various subsystems of functioning exist simultaneously, and mutually influencing one another. Harrison et al. has suggested that the stress of the motor system correlates with physiological stress indexed by oxygen saturation and heart rate $^{19)}$. These findings suggest that one part of the stress behavior of subsystems, such as autonomy, motor, and state system, affects stress behavior of the other subsystems. Therefore, stress behavior in one subsystem may influence the overall organization of behavioral development for of an infant.

The limitations of this study must be considered. The NBAS was utilized in this study to standardize handling for the assessment of infants' performances. However, the amount of stimulation was adjusted to support infants' behavior during the evaluation of the NBAS. As the NBAS is a structured examination with a preferred but not invariable order of administration, the examination sequence is modified in response to an infant's state changes, for example when a baby cries or becomes alert. However, in this study, it was the response to handling which was the focus of the behavioral observations, and the NBAS exam allowed opportunities over a period of time to handle the infant by positioning and other maneuvers. The handling is clinically salient for care providers, even though the magnitude of stress induced by handling cannot be predefined. Without adjusting the amount of stimulation according to the stress, the difference in the frequencies of stress behaviors between groups might become large. For the standardized observation of stress behavior, more analysis will necessary in future studies.

In conclusion, the with-PVL group showed significantly higher frequencies of stress behaviors in the neonatal behavior subsystems of autonomic, motor, and state-regulation responses than the without-PVL group. This finding suggests that the with-PVL group has characteristics of poorer motor control, less responsiveness to environmental stimuli, less regulatory capacity and poorer state regulation than the without-PVL group. Assessments of individualized behavioral signs provide us with useful information for understanding the development and adaptability of infants' behavioral organization. These neurobehavioral characteristics may result in increased risks of problems in the relationship between parents and child, as well as elevating the levels of parental depression, stress and anxiety. Furthermore, parents who have infants with these characteristics may experience more difficulties in handling, and interacting with, their babies. Thus, parents need help to modify their caregiving or parenting practices to adapt to there child's neurobehavioral characteristics, and early intervention should focus on enhancing parenting skills that are congruent with an infant's abilities.

\section{REFERENCES}

1) Stanley FJ: Survival and cerebral palsy in low birthweight infants: implications for perinatal care. Paediatr Perinat Epidemiol, 1992, 6: 298-310.

2) Kuban KC, Leviton A: Cerebral palsy. N Engl J Med, 1994, 330: 188-195.

3) Volpe JJ: Brain injury in the premature infant. Neuropathology, clinical aspects, pathogenesis, and prevention. Clin Perinatol, 1997, 27: 567-587.

4) Als H: A synactive model of neonatal behavioral organization: framework for the assessment and support of the neurobehavioral development of the premature infant and his parents in the environment of the neonatal intensive care unit. Phys Occup Ther Pediatrics, 1986, 6: 3-53.

5) Als H: Toward a synactive theory of development: promise for the assessment of infant individuality. Inf Mental Health J, 1982, 3: 229-243. 
6) Ohgi S, Akiyama T, Fukuda M: Neurobehavioural profile of low-birthweight infants with cystic periventricular leukomalacia. Dev Med Child Neurol, 2005, 47(4): 221-228.

7) Als H: Program Guide -Newborn Individualized Developmental Care and Assessment Program (NIDCAP): An Education and Training Program for Health Care Professionals. Boston: Children's Medical Center Corporation, 1996.

8) Ohgi S, Arisawa K, Takahashi T, et al.: Neonatal behavioral assessment scale as a predictor of later developmental disabilities of low birth-weight and/or premature infants. Brain Dev, 2003, 25(5): 313-321.

9) Brazelton TB, Nugent JK: Neonatal behavioral assessment scale, CDM, 3rd ed. Cambridge, MA: Cambridge University Press, 1995.

10) Pressler JL, Hepworth JT, Helm JM, et al.: Behaviors of very preterm neonates as documented using NIDCAP observations. Neonatal Netw, 2001, 20(8): 15-24.

11) DiPietro JA, Hodgson DM, Costigan KA, et al:: Development of fetal movement - fetal heart rate coupling from 20 weeks through term. Early Hum Dev, 1996, 44(2): 139-151.

12) Grunau RE, Holsti L, Whitfield MF, et al.: Are twitches, startles, and body movements pain indicators in extremely low birth weight infants? Clin J Pain, 2000, 16(1): 37-45.
13) Grunau RE, Oberlander $T$, Holsti L, et al.: Bedside application of the Neonatal Facial Coding System in pain assessment of premature neonates. Pain, 1998, 76(3): 277-286.

14) Prechtl HF, Einspieler C, Cioni G, et al.: An early marker for neurological deficits after perinatal brain lesions. Lancet, 1997, 349: 1361-1363.

15) Cioni G, Prechtl BY, Ferrari F, et al.: Comparison between observation of spontaneous movement and neurological examination in preterm infants. J Pediatr, 1997, 130: 704-711.

16) Bos AF, Einspieler C, Prechtl HF: Intrauterine growth retardation, general movements, and neurodevelopmental outcome: a review. Dev Med Child Neurol, 2001, 43: 61-68.

17) Guzzetta A, Mercuri E, Rapisardi G, et al.: General movements detect early signs of hemiplegia in term infants with neonatal cerebral infarction. Neuropediatrics, 2003, 34: 61-66.

18) Hadders-Algra M: General movements: a window for early identification of children at high risk for developmental disorders. J Pediatr, 2004, 145: S12S18.

19) Harrison LL, Roane C, Weaver M: The relationship between physiological and behavioral measures of stress in preterm infants. J Obstet Gynecol Neonatal Nurs, 2004, 33(2): 236-245. 